\title{
Budding yeast RNA polymerases I and II employ parallel mechanisms of transcriptional termination
}

\author{
Junya Kawauchi, ${ }^{1}$ Hannah Mischo, ${ }^{1}$ Priscilla Braglia, ${ }^{1}$ Ana Rondon, and Nick J. Proudfoot ${ }^{2}$ \\ Sir William Dunn School of Pathology, Oxford OX1 3RE, United Kingdom
}

\begin{abstract}
Both RNA polymerase I and II (Pol I and Pol II) in budding yeast employ a functionally homologous "torpedo-like" mechanism to promote transcriptional termination. For two well-defined Pol II-transcribed genes, CYC1 and PMA1, we demonstrate that both Rat1p exonuclease and Sen1p helicase are required for efficient termination by promoting degradation of the nascent transcript associated with Pol II, following mRNA 3' end processing. Similarly, Pol I termination relies on prior Rnt1p cleavage at the $3^{\prime}$ end of the pre-rRNA $35 \mathrm{~S}$ transcript. This is followed by the combined actions of Rat1p and Sen1p to degrade the Pol I-associated nascent transcript that consequently promote termination in the downstream rDNA spacer sequence. Our data suggest that the previously defined in vitro Pol I termination mechanism involving the action of the Reb1p DNA-binding factor to "road-block" Pol I transcription close to the termination region may have overlooked more complex in vivo molecular processes.
\end{abstract}

[Keywords: 5'-3' exonuclease; RNA polymerase I; S. cerevisiae; transcription termination]

Supplemental material is available at http://www.genesdev.org.

Received November 13, 2007; revised version accepted February 29, 2008.

Nuclear transcription in eukaryotes is performed by three different DNA-dependent RNA polymerases (Pol I, Pol II, and Pol III) resulting in the synthesis of ribosomal RNA (rRNA), messenger RNA (mRNA), and small noncoding RNAs (5S rRNA and tRNA). Other small RNAs (e.g., snRNAs) are synthesized by either Pol II or Pol III (Archambault and Friesen 1993). Knowledge of the molecular details that result in transcriptional initiation (e.g., through enhancer and promoter recognition) is now quite well advanced, especially for Pol II. However, the subsequent stages of the transcription cycle, elongation and termination, are less well described. In particular, the molecular process that switches each class of polymerase from processive elongation into termination is poorly understood but may differ significantly for each polymerase class. For Pol III, which copies relatively short genes, the mechanism of termination appears to be an intrinsic feature of the polymerase itself and especially the small subunit Rpc1lp. Here simple sensestrand oligo(dT) sequences appear to define Pol III termination sites (Braglia et al. 2005). In marked contrast, the mechanism of Pol II termination is far more complex, being intimately connected to processing of the premRNA. Thus capping, splicing, and cleavage/polyade-

\footnotetext{
${ }^{1}$ These authors contributed equally to this work.

${ }^{2}$ Corresponding author.

E-MAIL Nicholas.proudfoot@path.ox.ac.uk; FAX 44-1865-275556.

Article is online at http://www.genesdev.org/cgi/doi/10.1101/gad.463408.
}

nylation of the pre-mRNA all occur cotranscriptionally, mediated by mRNA processing factors that are known to interact directly or indirectly with the Pol II large subunit, the C-terminal domain (CTD) region, depending on its phosphorylation state (Orphanides and Reinberg 2002). Significantly, Pol II termination is dependent on pre-mRNA $3^{\prime}$ end processing and requires a specific set of termination factors and complex genetic signals (Proudfoot 2004; Buratowski 2005). Additionally, Pol II termination requires at least some of the cleavage/polyadenylation factors that, as well as processing the premRNA, may elicit structural or conformational changes to Pol II, somehow priming this molecular machine to terminate (Proudfoot 2004). Coupled to this, cleavage at the poly(A) signal generates a nascent RNA 3' product still attached to elongating Pol II. This RNA has an exposed, uncapped $5^{\prime}$ terminus that is now susceptible to nuclear $5^{\prime}-3^{\prime}$ exonuclease degradation by Saccharomyces cerevisiae Ratlp (Kim et al. 2004b) or mammalian Xrn2 (West et al. 2004). Several studies have shown that this degradation process results in removal of the nascent RNA up to the elongating Pol II that then induces polymerase template release. This termination process has been dubbed the "torpedo" mechanism (Tollervey 2004). However, the relative importance of nuclease "torpedoes" over cleavage/poly(A) factors in eliciting Pol II termination remains an issue of active research and debate in the field (Luo et al. 2006; Kaneko et al. 2007). A further complexity of the Pol II termination process is 
that some specialized small genes, such as those encoding snRNA and snoRNA, in S. cerevisiae operate an ostensibly different termination mechanism involving an RNA-binding factor, Nab3p; a CTD-binding factor, $\mathrm{Nrdlp}$; as well as a large protein containing a helicase domain called Sen1p (Steinmetz et al. 2001; Kim et al. 2006). It is possible that some or all of these factors may also act to promote termination on specific classes of Pol II genes encoding polyadenylated mRNAs (Steinmetz et al. 2006).

Pol I transcription displays many close parallels with that of Pol II. Transcription elongation, as with Pol II, is coupled to pre-rRNA processing. This can be visualized by EM analysis of rDNA captured by the "Miller Spreading" technique, which shows characteristic "Christmas tree" structures representative of multiple Pol I elongation complexes transcribing the rDNA template in tandem (French et al. 2003). Nascent pre-rRNAs display RNA:protein complex particles associated with the $5^{\prime}$ termini of progressively lengthening pre-rRNA, still attached to the Pol I:rDNA template. This complex contains RNA processing activities that cotranscriptionally cleave and degrade the transcript, thus generating mature rRNA (Dragon et al. 2002). The mechanism of Pol I transcriptional termination has also been investigated extensively, especially by using in vitro systems. In both yeast and mammals, a DNA-binding protein, Reblp in $S$. cerevisiae and TTF1 in mouse, is shown to interact with specific DNA sequences in the spacer sequence downstream from the Pol I transcription unit (Grummt et al. 1986; Kuhn et al. 1990; Lang and Reeder 1993). This DNA-bound protein was shown to pause Pol I elongation, which then promoted transcriptional termination at a pyrimidine-rich "release" sequence just upstream of the terminator-bound Reblp (Lang et al. 1994; Jeong et al. 1995). In mammals, Pol I transcript release at this position is facilitated by a specific release factor (Jansa et al. 1998; Jansa and Grummt 1999). While this mechanism may account for Pol I termination in purified in vitro systems, the in vivo picture is more complex. Several additional Pol I termination factors have been described more recently that suggest that the Pol I termination mechanism may be more complex than anticipated. In particular, the inessential Rpa12p small subunit of Pol I is required for both Pol I elongation and termination based on transcription run on (TRO) analysis of an rpa12 gene deletion strain (Prescott et al. 2004). This suggests a direct involvement of Pol I itself in the termination process. The endoribonuclease III protein Rntlp, which is known to generate the initial $3^{\prime}$ end cleavage of the prerRNA, is also required for efficient Pol I termination (Prescott et al. 2004). The requirement of Rntlp for Pol I termination points to the possibility that a $5^{\prime}-3^{\prime}$ exonuclease degradation process may act on the remaining nascent transcript, still attached to Pol I, and so promote termination by a torpedo-like mechanism.

We have re-evaluated the torpedo mechanism for Pol II termination and tested if Pol I also employs a related mechanism. While we confirm that both Rpa12p and Rntlp are indeed required for Pol I termination, we also show that Ratlp $5^{\prime}-3^{\prime}$ exonuclease is required. However, for both Pol II and Pol I termination, inactivation of Ratlp alone is insufficient to display a full termination defect. Instead, by combining Ratlp and Sen $1 p$ inactivation, we observe more severe termination defects. These data therefore point to the cooperation of these proteins in mediating efficient Pol II and Pol I termination. Consequently, we predict that each polymerase uses a torpedo mechanism to define the end of its transcription cycle.

\section{Results}

\section{Pol I termination factors}

We began these studies by re-evaluating the roles of previously defined Pol I termination factors. The well-documented involvement of Reblp was first tested by measuring its occupancy on chromatin associated with $S$. cerevisiae Pol I and Pol II promoters, as well as the Pol I termination region. Chromatin immunoprecipitation (ChIP) experiments were performed (Fig. 1A,B) on strains containing either genetically HA-tagged Pol I Rpa14p subunit or Reblp. Strong ChIP signals (Fig. 1A,B, gray bars) were detected across the Pol I 35S gene for the Rpa14p-tagged strain, but were at near background levels upstream of the Pol I promoter (Fig. 1A,B, US 5S primers) as well as downstream from the well-defined Pol I terminator region (Fig. 1A,B, cf. primer signals $2+3$ and 4 , $6+7)$. As expected, no significant signal was detected for the SIN3 Pol II promoter. In contrast, strong Reb1p signals (Fig. 1B, black bars) were detected over known Reblp-binding site regions in the Pol I rDNA and Pol II SIN3 promoters. Significantly, only background signals were detected in the Pol I terminator region (Fig. 1A,B, primers $2+3$ ), even though a Reblp-binding site has been defined in this region at least in vitro (Kulkens et al. 1989; Morrow et al. 1989). We therefore predict that while Reblp may associate transiently with the Pol I terminator region in such a manner that it is not detectable by ChIP, more stable interactions as observed for the rDNA and SIN3 promoters do not occur with chromatin at this position. To rule out potential artifact effects of the HA tag on Reblp binding or epitope masking, we repeated the ChIP analysis using a Reblp polyclonal antibody. However, again the ChIP signal was only detected over the Pol I promoter but not the terminator region (data not shown).

In spite of the lack of detectable Reblp on chromatin over the Pol I termination region, we elected to further investigate the requirement of both Reblp and its binding site region for Pol I termination. We initially carried out TRO analysis on a previously described yeast strain (Wai et al. 2001) containing engineered rDNA repeats that carry a 190-base-pair (bp) deletion in the Pol I termination region extending from just upstream of the Reblp-binding site to downstream from a T-rich sequence region, including both a binding site for a second DNA-binding protein (Reb2p) (Morrow et al. 1989) and a so-called fail-safe terminator region (Reeder et al. 1999). Since URA3 was inserted into this deletion (to allow its 
Kawauchi et al.

A

Figure 1. Role of Reb1p, Rpa12p, and Rnt1p in Pol I transcription termination. (A) Diagram of the rDNA repeat and the Pol II-transcribed locus SIN3. The primary rRNA transcript is indicated by a dashed line. The external transcribed sequence (ETS) and internal (ITS) transcribed sequence are shown. The triangle indicates the 3 ' cleavage site by Rntlp. Vertical arrows to the left and right of the Reblpbinding site (Reb1BS) denote the +93 primary termination site and the +250 fail-safe termination site, respectively. The black oval denotes the autonomously replicating sequence (ARS). The URA3 cassette (shown above) is inserted in place of the indicated terminator region of all rDNA repeats in strain TAK401. Horizontal bars denote the location of the primers and probes used in $\operatorname{ChIP}(B)$ and TRO $(C, D)$ analyses. Exact locations and sizes of the PCR products are described in Supplemental Table 3. (B) ChIP analysis (using anti-HA) of Pol I and Reblp binding across the Pol I-transcribed rDNA loci and SIN3 promoter. High Reblp signals are evident on the promoter Reb1-binding sites on rDNA and SIN3 promoter, but only background signal is detected on the Reb1 terminator site. Quantification of real-time PCR data was carried out as detailed in the Materials and Methods. All of the ChIP analyses were repeated $(n=2-4)$, and average data sets are presented. Error bars show SD throughout. $(C)$ Deletion of the Reb1BS-containing rDNA terminator impairs transcription termination. TRO hybridization profiles are presented for the terminator $\Delta$ strain (TAK401) and isogenic wild type (WT) (TAK314). Location of M13 or oligonucleotide probes are shown in $A$. Probes $3^{\prime}, 4^{\prime}$, and $5^{\prime}$ cover the URA3 sequence in TAK401, the same distance from the end of $35 \mathrm{~S}$ as corresponding probes 3, 4, and 5. (A) Actin-positive control; (M) M13-negative control (lacking rDNA sequence). Signals are corrected for the A content of each probe (TRO labeled with ${ }^{32} \mathrm{P}-\mathrm{UTP}$ ), and relative values against probe 2 are shown in the right panel. (D) Loss of Rpa12 or Rnt1 diminishes Pol I transcription termination. TRO hybridization profiles are presented for the rpa12 $\Delta$ and $r n t 1 \Delta$ strains compared with isogenic parental strains. Locations of M13 probes are shown in $A$. Quantification and controls are the same as in $C$.

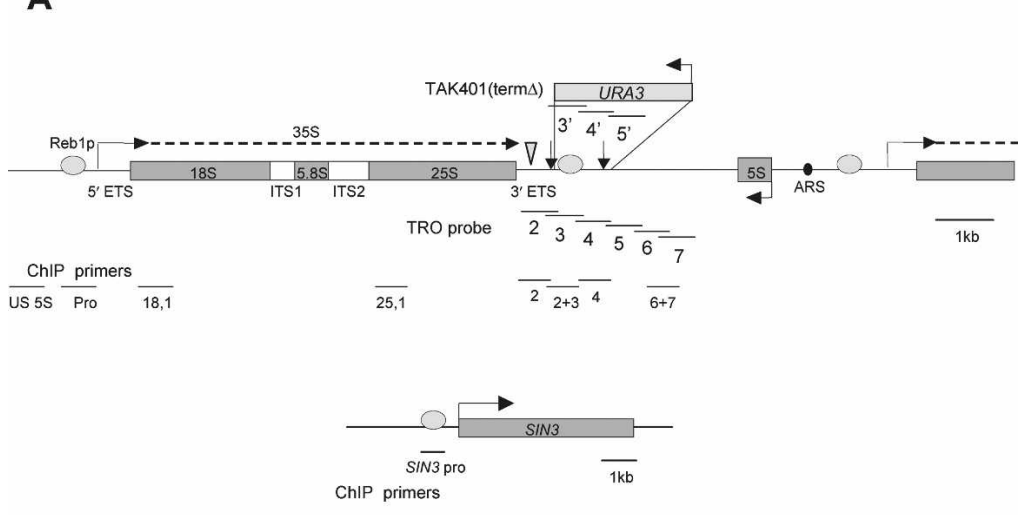

B ChlP

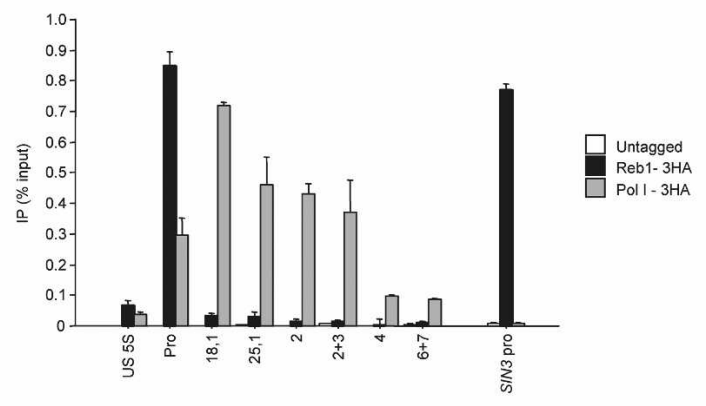

C TRO
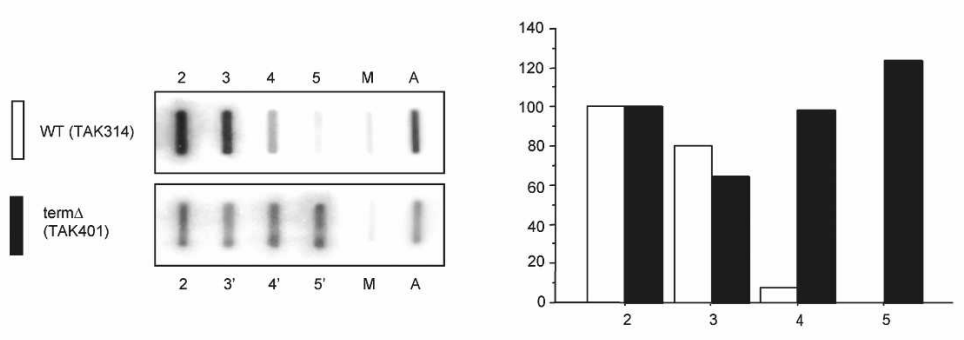

D TRO
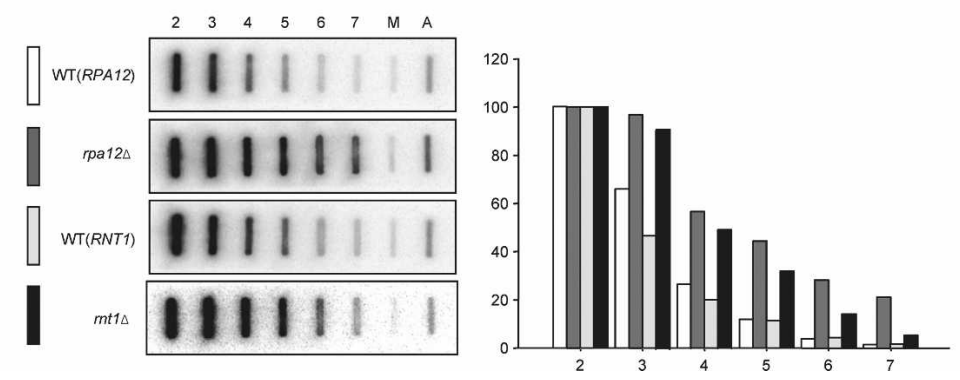

selection), we used TRO oligonucleotide probes $\left(3^{\prime}, 4^{\prime}\right.$, and $\left.5^{\prime}\right)$ over URA3 to detect read-through Pol I transcripts (Fig. 1A). As shown with the isogenic wild-type strain (TAK314), Pol I termination occurs efficiently beyond the Reblp-binding site region (Fig. 1C). However, in the deletion stain (TAK401), Pol I transcription ex- 
tends into the antisense URA3 sequences, indicating a disruption to the Pol I termination process (Fig. 1C). These data confirm that the Pol I terminator region is indeed required for efficient Pol I termination. We finally attempted to address whether Reblp itself is required for this termination process as predicted by in vitro experiments (Lang et al. 1994). We therefore engineered a yeast strain in which $R E B 1$ is tagged with a degron sequence to promote specific degradation of this protein when raised to a nonpermissive temperature (Supplemental Fig. 1A; Dohmen et al. 1994). However, even after $3 \mathrm{~h}$ of temperature shift, $\sim 25 \%$ of the Reblp, as compared with the amount of protein detected at the permissive temperature, was still detected in this strain. This suggests that strong selection acts to maintain minimal levels of this essential, pleiotropic transcription factor. TRO analysis of the Reb1p degron strain (showing fourfold reduction in Reblp levels) did not reveal any significant loss of Pol I termination as compared with wild-type cells (Supplemental Fig. 1B).

Overall, these data suggest that Reblp is unlikely to play a critical role in Pol I termination in vivo. Thus the absence of detectable Reblp on Pol I terminator chromatin as well as the lack of any detectable termination defect of a fourfold reduction in Reblp levels argues that the previous Pol I in vitro termination assays, which implicated Reb1p in this process (Lang and Reeder 1993; Lang et al. 1994), may have overlooked additional in vivo effects. However, it is also clear that the Pol I terminator sequence is required for Pol I termination to occur efficiently. Since this sequence is highly T-rich in the sense strand, it is possible that U-rich sequences as part of the nascent transcript in association with proteins bound to the template DNA may induce transcriptional pausing effects that could facilitate Pol I termination.

We next performed TRO analysis on strains deleted for either RPA12 or RNT1 (Fig. 1D). This shows a clear termination defect for both of these deletion mutant strains as compared with their isogenic wild types. While a strong termination defect for rpa12 $\Delta$ was reported previously, the defect observed for the $r n t 1 \Delta$ strain was found previously to be less severe (Prescott et al. 2004). However, we note that in our current analysis, loss of Rntlp appears to have almost as strong a termination defect as loss of Rpa12p. We predict that this experimental difference may reflect different growth conditions between these separate experiments.

Overall, these initial results confirm that both Rntlp and Rpa12p play significant roles in Pol I termination. However, our experiments on the role of Reblp in Pol I termination suggest that additional mechanisms are likely to operate in an in vivo situation.

\section{Both Rat1p and Sen1p are required for Pol II termination at the CYC1 and PMA1 genes}

Previous studies in yeast and mammals have demonstrated the involvement of $5^{\prime}-3^{\prime}$ exonucleases in Pol II termination of genes encoding polyadenylated mRNA. However, in these studies, the effect of deleting RAT1 on
Pol II termination was often relatively minor, causing only twofold to threefold read-through of Pol II beyond the normal site of termination (Kim et al. 2004b). We therefore sought to identify other factors that might cooperate with Ratlp in the Pol II termination process. An attractive candidate was the DEAD-box helicase Sen $1 p$ (Ursic et al. 1997), since this protein is a known component of the alternative Pol II termination pathway also involving the RNA-binding protein Nab3p and Pol II CTD-binding protein Nrdlp (Steinmetz et al. 2001). It seemed plausible that Sen1p, through its RNA helicase activity, could act to expose the downstream RNA product generated by cleavage and polyadenylation and so facilitate Rat1p-mediated degradation of the nascent, Pol II-associated transcript.

In these experiments, we initially used the well-defined Pol II terminator of the CYC1 gene (Birse et al. 1998). Yeast strains were generated with either single or double conditional (temperature-sensitive) mutations in RAT1 and SEN1, and each was transformed with a GAL1-CYC1-containing plasmid (Birse et al. 1998). TRO was performed on these four strains (wild type, rat1-1, sen1-1, and rat1-1 sen1-1) using a series of singlestranded M13 probes extending over and $3^{\prime}$ to the CYC1 gene (Fig. 2A). Note that the convergent gene UTR1 is not transcribed in this plasmid as its promoter is absent. Also, the endogenous CYC1-UTR1 locus is expressed at very low levels (Alen et al. 2002) and so does not significantly contribute to the observed run on signals. Figure 2B shows representative TRO data for the four yeast strains, obtained by probing over the plasmid-borne CYC1 gene. Quantitation of these TRO data is shown below by normalizing to the value obtained for probe 1 within the CYC1 gene. While wild type gives the expected strong termination pattern indicated by high signals for probes 1 and 2, lower signal for probe 3 and background signals for probes $4-6$, both the single mutant strains rat1-1 and sen 1-1, show similar levels of increased read-through transcription. Importantly the combined rat1-1 sen1-1 strain gave higher read-through transcription levels, indicating that the double mutation has an additive effect over the single mutations. These data were reproduced in multiple, independent experiments and gave closely similar transcription profiles.

To extend this analysis to a larger chromosomal gene, we carried out parallel experiments on the endogenous PMA1 gene. Unlike CYC1, this gene is relatively long $(2.7 \mathrm{~kb})$ and positioned upstream of tandem-orientated LEU1 (Fig. 3A). The lower levels of transcription obtained for this gene as compared with CYC1 (driven by the strong GAL1 promoter and on a multicopy plasmid) precluded the use of TRO analysis in this case. Instead, we performed ChIP analysis using Pol II antibody coupled with the detection of read-through transcripts by real-time PCR. As expected, in the wild-type strain, Pol II association with PMA1 chromatin declined to low levels following the gene's second poly(A) site, reflecting efficient Pol II termination (Fig. 3B, cf. ChIP signals for p5, p6 and p7, p9) (Kim et al. 2004a). In both the single rat1-1 and sen 1-1 mutant strains, significantly more Pol 
A

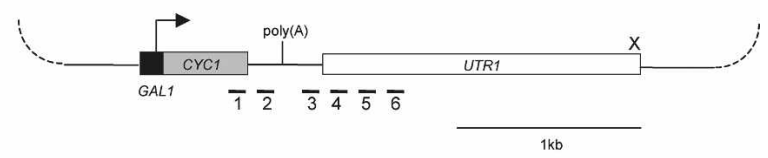

B TRO
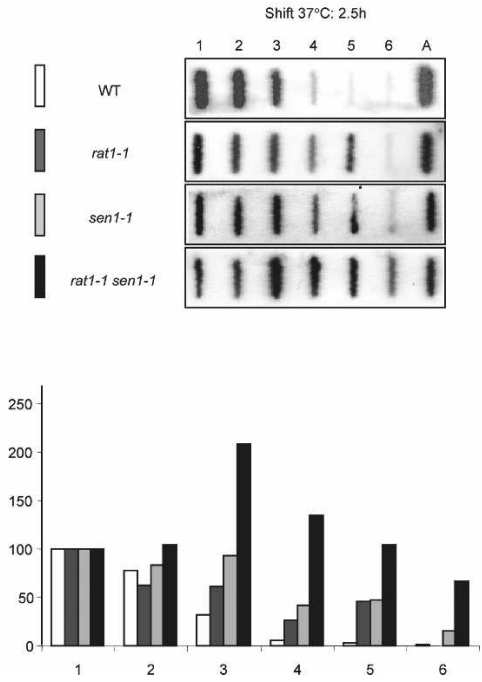

Figure 2. Rat $1 \mathrm{p}$ and Sen $1 \mathrm{p}$ are both required for efficient Pol II termination at the CYC1 locus. (A) Diagram of the pGCYC1 plasmid transformed into each of the indicated strains. The CYC1 gene promoter is replaced by that of GAL1, and the UTR1 promoter is deleted (cross). M13 probes used in TRO analysis are indicated by horizontal bars. The position of the CYC1 poly(A) site is indicated. (B) CYC1 gene TRO profiles for pGCYC1-transformed rat1-1, sen1-1, rat1-1 sen1-1, and isogenic wild-type strains. Quantitation of the TRO experiments is shown below. Values are plotted relative to probe 1, allowing direct comparison between the different strains.

II signal was detected with PCR primers $\mathrm{p} 7$ and $\mathrm{p}$ 9, indicative of Pol II termination defects. We also observe with rat1-1 that there appears to be some depletion of Pol II signal over the 3' end of the gene (p5 and p6), which may reflect enhanced pausing in this mutant background. Strikingly, with the double rat1-1 sen1-1 strain, even higher levels of Pol II were detectable downstream from the normal termination region. We note that a small increase in Pol II occupancy is detected for the inactive negative control gene MUC1 in both the sen1-1 and rat1-1 sen 1-1 mutant strains (Fig. 3B). This may reflect read-through transcription from adjacent gene loci. The RT-PCR analysis of PMA1 read-through transcripts with the same four strains showed broadly similar results (Fig. 3C). While the wild-type strain gave little detectable signal beyond the second poly(A) signal, both rat1-1 and the double rat1-1 sen1-1 strains gave high levels of read-through transcripts reading into downstream LEU1. The sen1-1 strain gave lower, though significant, levels of read-through transcript. Since this RTPCR analysis measures total cellular RNA levels, we predict that inactivation of Ratlp will increase readthrough transcript levels both through loss of transcript degradation as well as loss of Pol II termination. Our results on PMA1 in these mutant strains broadly matches those obtained with CYC1. We therefore conclude that Ratlp and Sen $1 p$ cooperate to promote efficient Pol II termination on both genes.

\section{Pol I-transcribed rDNA is associated with Rat1p and $\operatorname{Sen} 1 p$}

Both Rat $1 p$ and Sen $1 p$ are required for fully efficient Pol II termination, at least for the two tested Pol II genes,

A

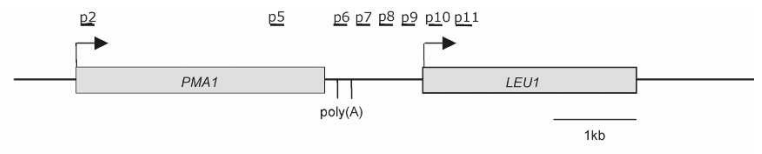

B $\operatorname{ChIP}(\mathrm{Pol}$ II)
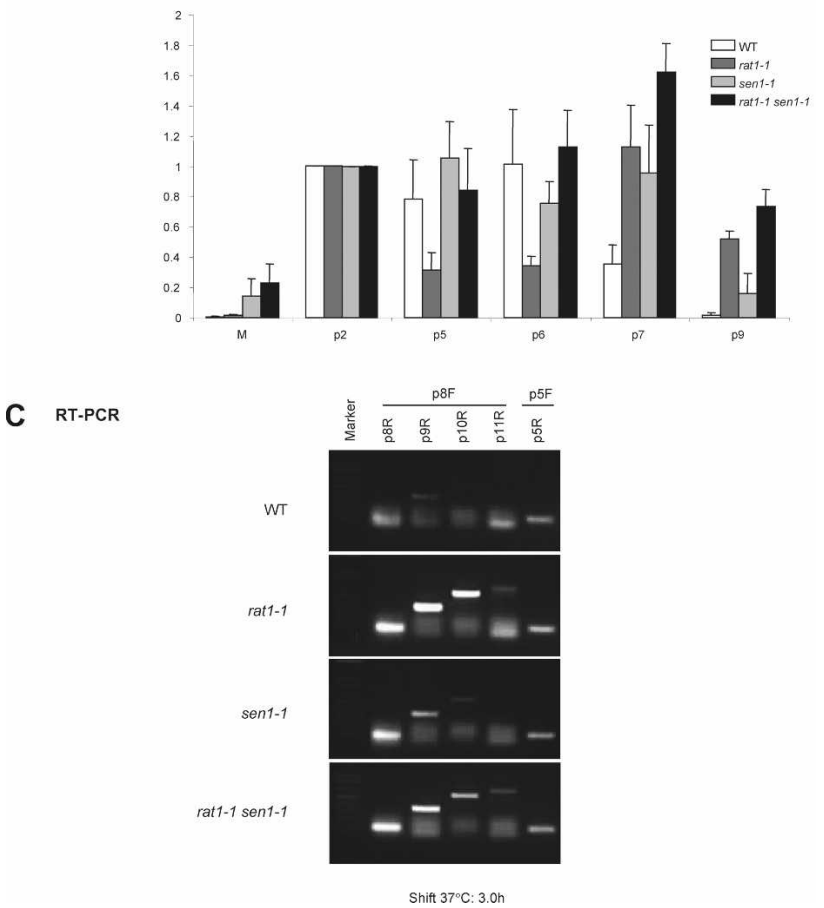

Figure 3. Role of Rat $1 \mathrm{p}$ and Sen $1 \mathrm{p}$ in transcription termination at the PMA1 locus. (A) Diagram of PMA1 and the following $L E U 1$ genes. Two alternative poly(A) sites of PMA1 are indicated. Horizontal bars denote the location of primers used in the ChIP $(B)$ and RT-PCR $(C)$ analyses. (B) Pol II ChIP over the PMA1 terminator region. Cells from the rat1-1, sen1-1, and rat1-1 sen1-1 strains or from the isogenic wild type were harvested $2.5 \mathrm{~h}$ after shift at $37^{\circ} \mathrm{C}$. ChIP values are expressed relative to the signal obtained with oligos p2 (see $A$ ). (M) The inactive $M U C 1$ gene, used as a negative control. $(C)$ RT-PCR analysis of transcript downstream from the PMA1 poly(A) sites. Total RNA was reverse-transcribed with specific RT primers (p5R, $\mathrm{p} 8 \mathrm{R}, \mathrm{p} 9 \mathrm{R}, \mathrm{p} 10 \mathrm{R}$, and $\mathrm{p} 11 \mathrm{R}$ ) and PCR-amplified with the primers indicated. After $3 \mathrm{~h}$ at $37^{\circ} \mathrm{C}$, the transcripts downstream from the poly(A) are stabilized in the rat1-1, sen1-1, and rat1-1 sen1-1 mutant strains with respect to the wild type. The lower bands seen in the $\mathrm{p} 9 \mathrm{R}, \mathrm{p} 10 \mathrm{R}$, and $\mathrm{p} 11 \mathrm{R}$ lanes are unused primer dimers. 
CYC1 and PMA1 (Figs. 2, 3). We therefore sought to establish a potential role for these two proteins in Pol I termination. As a first approach, we wished to determine if Ratlp and Sen $1 p$ are detectable on rDNA chromatin. We therefore used yeast strains with TAP tags on their endogenous RAT1 or SEN1 genes. We then performed ChIP analysis for the presence of Rat1p and Sen $1 \mathrm{p}$ by immuno-selecting TAP-associated chromatin and clearly detected the presence of both proteins across rDNA genes (Fig. 4A,B). We presume that Ratlp and Sen $1 p$ are associated with active rDNA repeats, as the silenced rDNA repeats are likely to be inaccessible. Rat1p appears to display some signal across the whole locus but with a signal peak over region 4 , just beyond the normal position of Pol I termination. Similarly, Sen $1 \mathrm{p}$ is detectable across the rDNA cluster with a peak of signal directly over the termination region. As a control, Rat $1 p$ and Sen $1 p$ were also detected over the CYC1 gene, and here again Ratlp gave a strong 3 ' but relatively weak 5' signal, while Sen 1 p gave more even, positive signals over both tested CYC1 regions. We conclude from these ChIP analyses that Ratlp and Sen1p are indeed associated with rDNA chromatin in such a manner that they may potentially play direct roles in Pol I transcription elongation and/or termination. In the case of Sen 1p, previous reports have noted the widespread association of Sen1p with Pol II genes consistent with our present data (Steinmetz et al. 2006).

Rat1p and Sen1p are detectable on rDNA chromatin, and both of these activities play a clear role in Pol II termination (Figs. 2, 3). We therefore measured total cellular rRNA transcript levels downstream from the Pol I termination region using RT-PCR analysis with strandspecific RT primers on RNA extracted from the wildtype, rat1-1, sen1-1, and rat1-1 sen1-1 strains (Fig. 4C). cDNA samples from each of these strains gave equivalent signals when amplified with primers $\mathrm{p} 0$ and $\mathrm{p} 1$, as some stable pre-rRNA is known to extend to this region. p0 is positioned immediately downstream from the Rntlp cleavage site (Fig. 4A). However, using further downstream primers (p2 and p3) extending into the Pol I spacer sequence, $3^{\prime}$ to the normal Pol I terminator with the common forward p0 primer, virtually no RT-PCR signal was obtained for wild-type RNA, while significant though declining signals were detected in the different mutant strains. These results demonstrate that normally

A

C RT-PCR

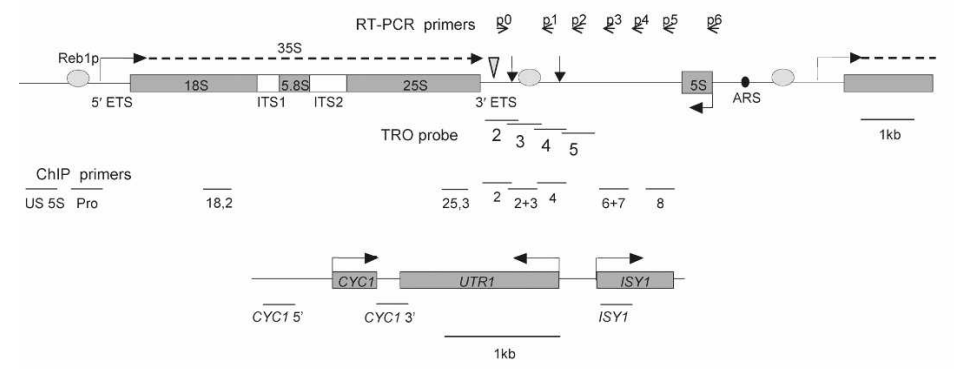

B ChIP
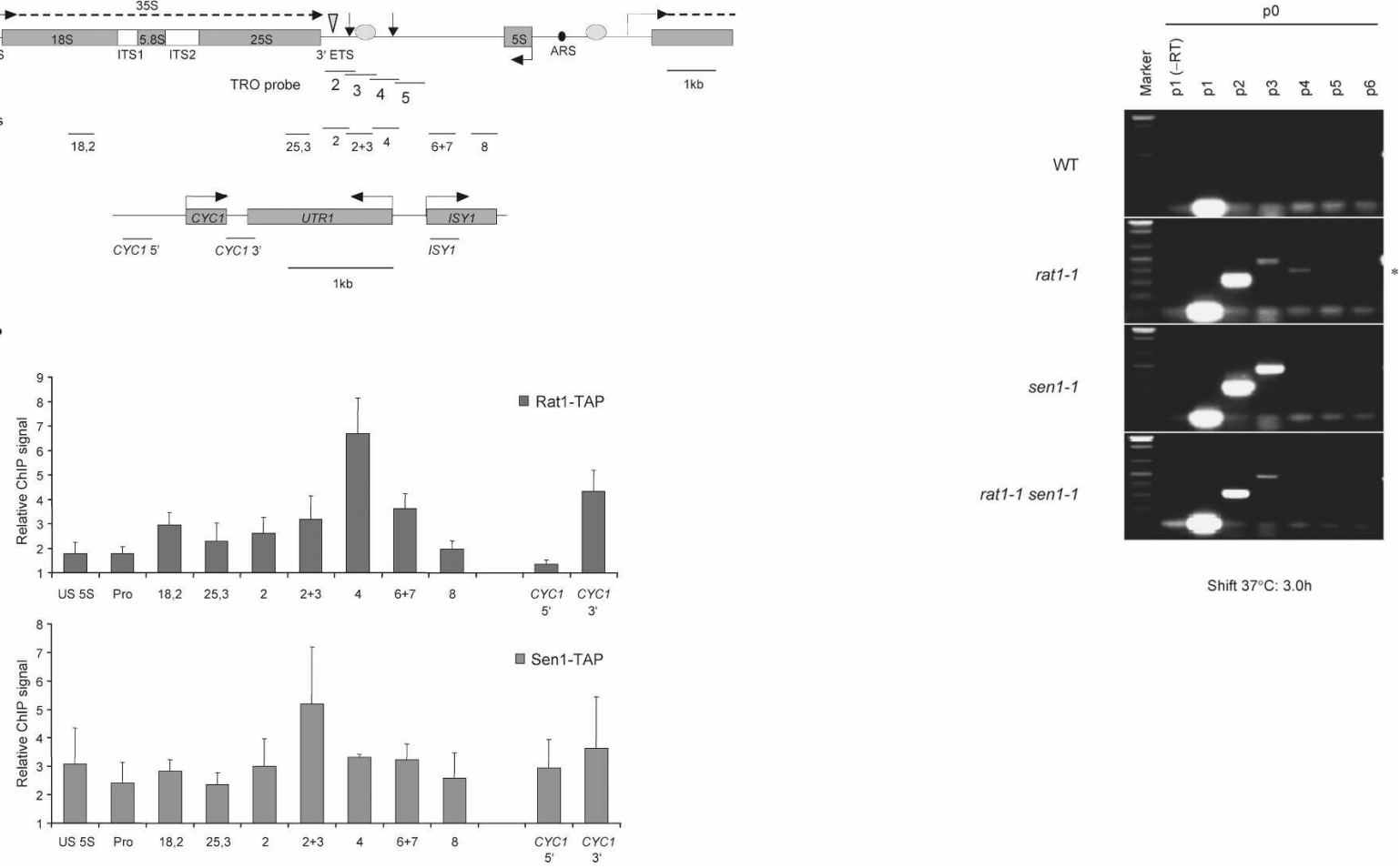

Figure 4. Rat $1 \mathrm{p}$ and Sen $1 \mathrm{p}$ are associated with rDNA chromatin and cooperatively degrade the $3^{\prime}$ product of Rntlp cleavage. $(A)$ Location of the primers and probes used in ChIP, RT-PCR, and TRO analyses. A map of an rDNA repeat is shown as in Figure 1A with the exact positions of the probes and primers described in Supplemental Table 3. (B) ChIP analysis of TAP-Ratlp and TAP-Sen1p yeast strains. Both the proteins associate with rDNA chromatin accumulating over the terminator region. The signals obtained are expressed relative to the isogenic nontagged strain. Pol II-transcribed CYC1 gene is shown as a control. (C) RT-PCR analysis of transcript downstream from the Rnt1p cleavage site. Extracted total RNA was reverse-transcribed with strand-specific RT primers (p1-p6) and PCR-amplified with the primer set shown in $A$. After $3 \mathrm{~h}$ at $37^{\circ} \mathrm{C}$, the transcript downstream from the Rntlp site is stabilized in the rat1-1 and/or sen1-1 strains. The asterisk on the right indicates a nonspecific PCR product. Lower bands are unused primer dimers. 
unstable spacer transcripts can be stabilized by inactivation of Ratlp and/or Senlp. They therefore point to the possibility that the $3^{\prime}$ product of Rntlp cleavage is susceptible to degradation directly or indirectly by these RNA-modifying enzymes. Since deletion of RNT1 causes a Pol I termination defect, our results in combination point to a torpedo-like mechanism for Pol I termination, as ascribed to Pol II termination.

\section{Rat1p and Sen1p cooperate to promote efficient Pol I termination on rDNA genes}

We next measured the profile of actively transcribing Pol I across rDNA genes in the wild-type, rat1-1, sen 1-1, and rat1-1 sen1-1 strains using TRO analysis, and then confirmed these results by Pol I ChIP analysis. Each of these strains were subjected to a time course of growth at the restrictive temperature of $37^{\circ} \mathrm{C}$ and then analyzed by TRO. Single-stranded M13 probes 2-5 (Fig. 1A) across the Pol I termination region were used in this analysis. As shown in Figure 5A, while a slight increase in TRO signal over probes 4 and 5 was observed with the rat1-1 sen 1-1 strain at $2.5 \mathrm{~h}$ of restrictive growth, a significant termination defect was visible at $3.5 \mathrm{~h}$. Thus, threefold higher signal than wild type was detectable over downstream regions detected by probes 4 and 5 . At the longest time point of $5 \mathrm{~h}$, the single mutant rat1-1 strain also displayed a modest termination defect. In contrast, the double rat1-1 sen1-1 strain showed a somewhat reduced termination defect at this later time point, suggesting that alternative Pol I termination mechanisms become more significant.

These TRO analyses were validated by performing Pol I ChIP analysis (Fig. 5B) on all three mutant strain versus wild type, again using a time course of growth at the restrictive temperature. A clear increase in Pol I signal was detectable at the 3 -h time point using spacer primers downstream from the Pol I termination region for the double rat1-1 sen1-1 mutant strain. Also, some increase in Pol I signal was detected, especially with primers $6+7$ for the single rat1-1 mutant. These effects were maintained, although again at a slightly reduced level, for the longer 5-h time point of restrictive growth. The Pol II-transcribed gene ISY1 is also shown as a negative control.

Overall, our data demonstrate that Rat $1 p$ and Sen $1 p$ in combination play a significant role in promoting efficient Pol I termination, just as observed for Pol II termination. We therefore predict that efficient Pol I termination requires a combination of the previously described Reblp-mediated termination mechanism as well as a torpedo mechanism.
Figure 5. Rat 1 and Sen 1 are involved in Pol I transcription termination. (A) TRO analysis of rat1-1 and/or sen 1-1 strains. Cells were harvested at three different time points $(2.5,3.5$, and $5.0 \mathrm{~h})$ after shift at $37^{\circ} \mathrm{C}$. The M13 probes are indicated in Figure 4A. Signal intensity was normalized to probe 2 , and quantification of the profiles obtained is shown in the bottom panel. The top panel shows a representative TRO hybridization profile $(3.5 \mathrm{~h}$ after temperature shift). (B) Pol I occupancy over the terminator region is increased in rat1-1 and rat1-1 sen1-1 mutants. ChIP analysis was conducted with 3HAPol I strains (Rpa14p subunit). Cells from the rat1-1, sen1-1, rat1-1 sen1-1 mutant strains or from the isogenic wild-type strain were harvested 0,3 , or $5 \mathrm{~h}$ after temperature shift. ChIP values are expressed relative to the signal obtained with oligos 25,3 (see Fig. 4A). The Pol II-transcribed ISY1 gene is shown as a negative control.
A TRO
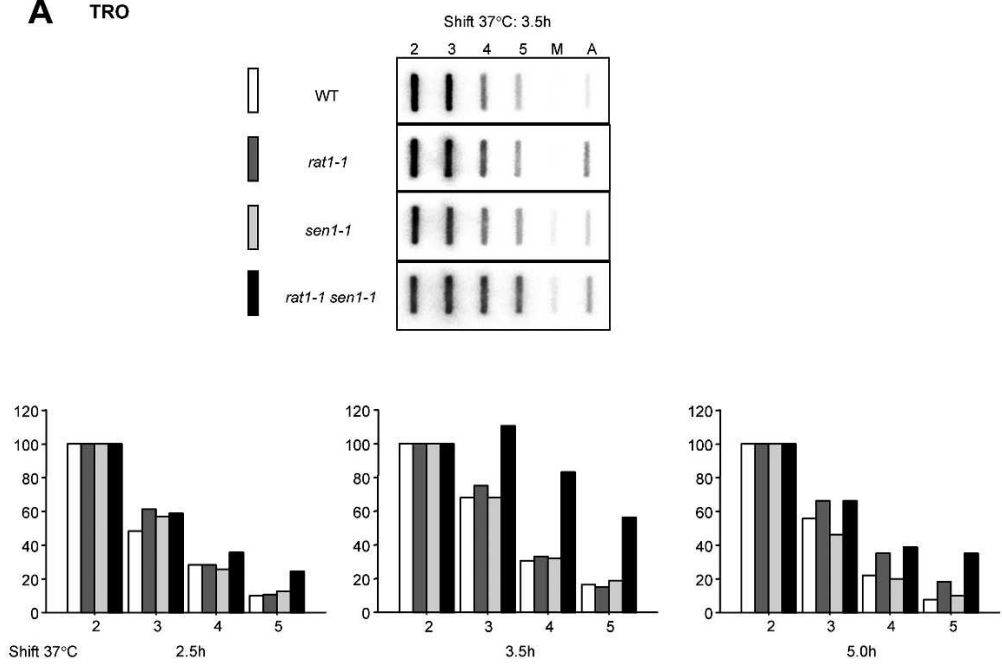

B ChIP (Pol I)

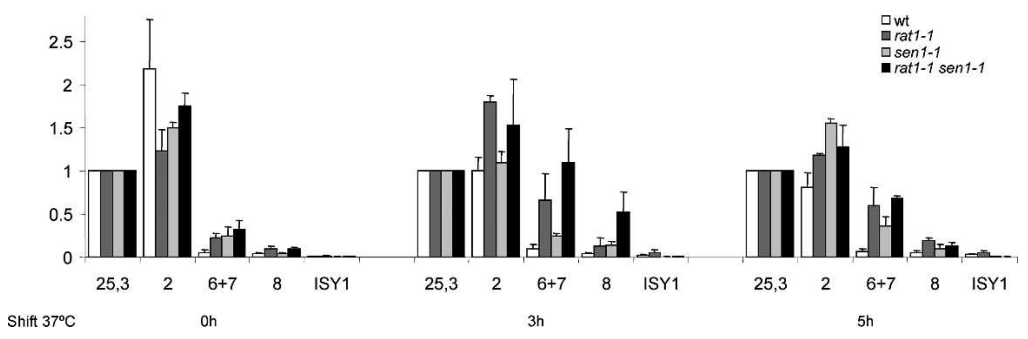


rDNA spacer sequence Pol II transcripts do not influence Pol I termination

Two recent papers (Houseley et al. 2007; Vasiljeva et al. 2008) describe the presence of cryptic unstable transcripts (CUTs) transcribed by Pol II in the rDNA spacer region. These CUTs are detected readily only by inactivation of the exosome RNA degradation complex. One of these CUTs in particular is antisense to rRNA and is transcribed toward the Pol I gene, ending near the Pol I termination region. Furthermore, as with other yeast CUTs, the mechanism of Pol II termination employs the Nrd1p/Nab3p/Sen1p complex (Arigo et al. 2006; Vasiljeva et al. 2008). We wished to determine if this Pol II CUT that is close to the Pol I termination region plays a role in the Pol I torpedo-mediated termination mechanism. To achieve this, we selectively inhibited Pol II but not Pol I transcription during the TRO reaction by treating permeabilized cells with $\alpha$-amanitin. As shown in Figure 6A, we demonstrate that both the wild-type and mutant (rat1-1 sen1-1) TRO profiles on rDNA are unchanged with or without $\alpha$-amanitin treatment. Also, the strong reduction in Pol II TRO signal over the Pol II-transcribed gene (Fig. 6A, probe K1) following $\alpha$-amanitin treatment confirms that this drug treatment was effective in inhibiting Pol II.

The levels of rDNA spacer CUTs are thought to be restricted not only by exosome-mediated degradation but also by SIR2-dependent chromatin silencing. Consequently, inactivation of Sir2p results in a significant increase in the levels of rDNA spacer CUT synthesis (Houseley et al. 2007; Vasiljeva et al. 2008). We therefore reasoned that while inhibition of CUT synthesis by $\alpha$ - amanitin treatment did not affect Pol I termination, the opposite effect of increasing CUT levels could result in some Pol I termination defect. However, as shown in Figure 6B, TRO analysis of the Pol I termination region revealed no detectable loss of Pol I termination efficiency with or without Sir2p.

Overall, our analysis of the possible interplay between the Pol II-derived CUT and the adjacent Pol I termination region indicates that these two transcription units do not influence each other.

\section{Discussion}

The results presented in this study suggest that the mechanisms of Pol I and Pol II termination display strong parallels, as predicted previously (Prescott et al. 2004). In particular, both polymerase systems rely on a torpedo-like mechanism to bring about efficient termination. It has been suggested (Kufel et al. 1999) that Rnt1p cleavage downstream from the 35S RNA transcript is the initial defining step for pre-rRNA processing that begins the complex series of RNA processing and modifying reactions that result in efficient $18 \mathrm{~S}, 5.8 \mathrm{~S}$, and $25 \mathrm{~S}$ rRNA synthesis. However, the mechanism of Pol I termination was predicted to be independent of these RNA processing reactions, depending solely on Reblpmediated pausing of elongating Pol I that in turn causes back-tracking of Pol I and its release by slippage on a close upstream pyrimidine-rich sequence (Lang et al. 1994). Our data now extend this simplistic model for Pol I termination to a mechanism that is coupled to both cleavage by Rntlp and cotranscriptional degradation of
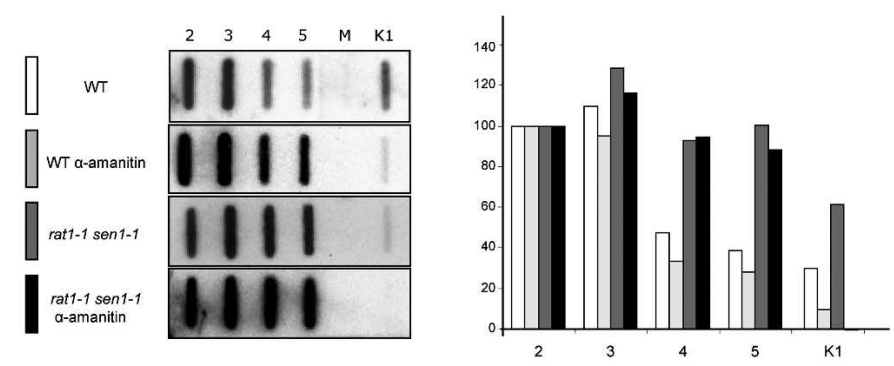

B TRO
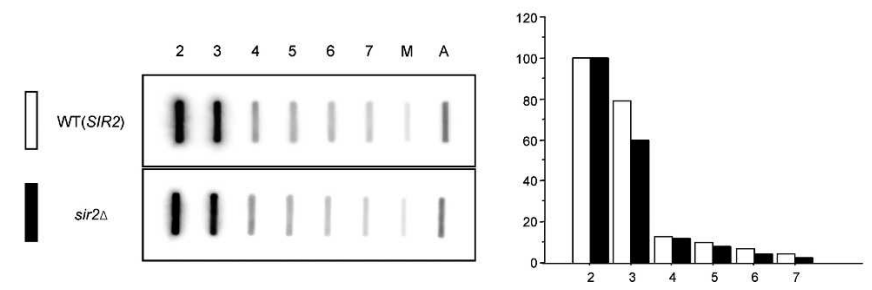

Figure 6. Pol I termination profile is not influenced by CUTs. (A) Inhibition of CUT synthesis does not influence Pol I transcription termination. pTEF:KAN ${ }^{R}$-transfected rat1-1 sen1-1 and wild-type strains were treated with $\alpha$-amanitin $(50 \mu \mathrm{g} / \mathrm{mL})$ before TRO reaction, and profiles were compared with those from untreated cells. Probes for Pol I transcription (2, 3, 4, and 5) are the same as in Figure 5A. Probe K1 shows the signal from pTEF: $K A N^{R}$, transcribed by Pol II. Note that Pol II transcription is repressed after $\alpha$-amanitin treatment. $(B)$ Sir2 deletion does not influence Pol I transcription termination. Probes are shown in Figure 1A. The TRO profile of the sir2s mutant strain has no significant difference from that obtained with the parental strain. 
Figure 7. "Torpedo" model for Pol I transcription termination. Nascent rRNA transcribed by Pol I is cleaved by Rntlp (gray scissors) cotranscriptionally. This releases the pre-rRNA for processing into mature rRNAs. $5^{\prime} \rightarrow 3^{\prime}$ exonuclease Ratlp is recruited to the $5^{\prime}$ end of the downstream Rntlp cleavage product. Here it degrades the Pol I-associated transcript assisted by the helicase activity of Senlp. Eventually, the Ratlp torpedo catches up with Pol I and induces termination.

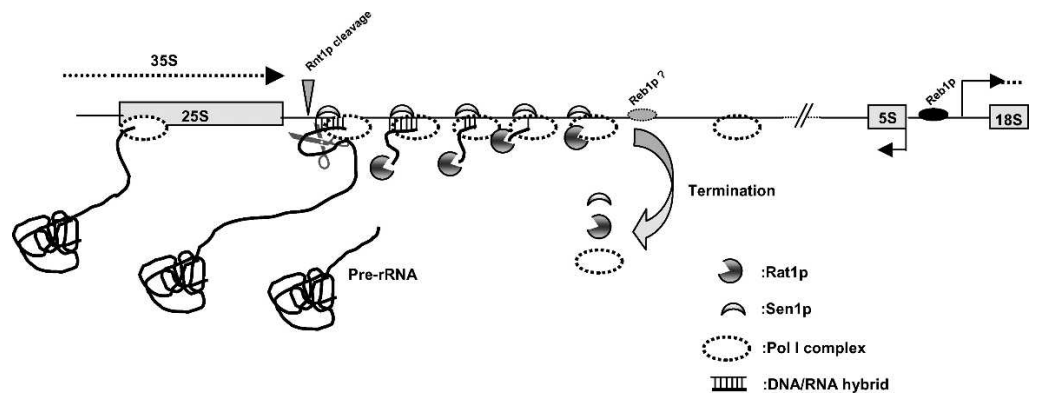

the $3^{\prime}$ transcript associated with Pol I still elongating into the rDNA spacer sequence (Fig. 7). In effect, we predict that both Pol I and Pol II employ a closely related torpedo mechanism to promote efficient polymerase release. Our new results do not exclude a role for Reblp in Pol I termination. Although we do not detect Reb1p on chromatin around the normal termination site, it is known that Reblp can specifically bind naked DNA at this site, since a conserved Reb1p consensus sequence is present here (Ganley et al. 2005) and clearly binds Reblp in vitro (Lang and Reeder 1993). We predict that in vivo on a chromatin template, Reblp interaction may be more transient. Indeed, we demonstrated recently a role for chromatin remodeling enzymes in Pol I termination (Jones et al. 2007), reflecting a need to alter chromatin structure over the termination region that may act to displace Reb1p from the template. A further structural complexity may relate to the presence of gene looping across the rDNA between promoter- and terminatorbound Reb1p. Such loops have been demonstrated in all tested Pol II-transcribed genes (O'Sullivan et al. 2004). Significantly, a recent analysis of murine rDNA demonstrates a loop conformation between the Pol I promoter and terminator sequences. Furthermore, the mouse homolog of Reb1p, TTF1, is implicated in the formation of this structure (Nemeth et al. 2008).

The observations in this study that both Pol I and Pol II termination mechanisms require not only Rat $1 p$ but also Sen $1 p$ to be fully efficient raise interesting mechanistic issues. Previously, Senlp has been shown to act with Nrd1p and Nab3p to promote termination on smaller Pol II transcription units such as snoRNA/snRNA genes, CUTs, and shorter mRNA-encoding genes (Steinmetz et al. 2001, 2006; Arigo et al. 2006). However, the clear requirement of Sen $1 p$ for PMA1 termination (Fig. 3) argues that longer Pol II-transcribed genes may also require this activity. Whether Sen $1 p$ acts together with Nrd1p and Nab3p in this case remains to be established. The fact that Pol I termination also requires the combined actions of Rat $1 p$ and Sen $1 p$ argues that Sen $1 p$ may play a separate role from Nrd1p/Nab3p in Pol I- and some Pol II-transcribed genes. This appears especially likely as Nrd1p/Nab3p is known to interact with the Pol II CTD domain, which is absent from Pol I (Kuhn et al. 2007). A further connection between Sen 1 p and Pol I termination comes from the observed interaction between Sen 1p and Rntlp (Ursic et al. 2004). We propose that Sen $1 p$ helicase activity may act directly with Rat1p to expose the nascent transcript following Rntlp cleavage [or poly(A) site cleavage] and so promotes more efficient Ratlp exonucleolytic degradation. Further in vitro analysis of Rat1p and Sen $1 p$ will be required to characterize this potential mechanism.

Finally, it is worth considering the extraordinary complexity of the rDNA gene spacer repeat unit. Within each unit, all three-Pol I, Pol II, and Pol III-generate transcripts, 35S pre-rRNA, 5S RNA, and CUTs. Added to this array of three different gene classes, rDNA spacer sequence also contains a replication origin, a mitotic cohesion site, and a sequence that controls rDNA repeat copy number. This remarkable concentration of critical sequence elements must necessitate a clear delineation between each functional unit. The necessity for efficient Pol I termination is therefore likely to be of paramount importance.

\section{Materials and methods}

\section{Yeast strains}

The strains used in this study are presented in Supplemental Table 1 .

FD-4D, FD-4C, FD-4B, FD-4A, and the relative Pol I-tagged strains (Rpa14-3HA) were grown in YPD or minimal media at $25^{\circ} \mathrm{C}$ and transferred to $37^{\circ} \mathrm{C}$ as indicated. The Reb1-HA-degron strain was grown in YPD in the presence of G418 $(200 \mu \mathrm{g} / \mathrm{mL})$ at $25^{\circ} \mathrm{C}$, then shifted at $37^{\circ} \mathrm{C}$ as indicated. All the other strains were grown in YPD at $30^{\circ} \mathrm{C}$.

\section{ChIP}

The strains used for ChIP analyses were AS14, BY4742, Reb1HA, rat1-1 Pol I-HA, sen1-1 Pol I-HA, rat1-1 sen1-1 Pol I-HA, wild-type Pol I-HA, wild-type SC0000, TAP-Rat1, and TAPSen1. Analysis was carried out as before (Jones et al. 2007) with the following modifications: The cross-linking time was $15 \mathrm{~min}$ (Fig. 1B) or $20 \mathrm{~min}$ (Fig. 4B), chromatin was sonicated using a Bioruptor, cross-links were reversed overnight with proteinase $\mathrm{K}$, and DNA was purified using Qiagen minicolumns. The antibodies used for ChIP analysis were Anti-HA clone F7 (Santa Cruz Biotechnology) in Figures 1B and 5B and Anti-polII CTD antibody 8WG16 (Covance) in Figure 3B.

The ChIP analysis in Figure 4B was conducted essentially as described (Keogh and Buratowski 2004) with the following modifications: Cross-linking was carried out for $20 \mathrm{~min}$ at $25^{\circ} \mathrm{C}$, and chromatin was sonicated using a Bioruptor. Immunoprecipitation (IP) was performed with IgG Sepharose 6 Fast Flow beads (Amersham), and elution was performed for $30 \mathrm{~min}$ at $65^{\circ} \mathrm{C}$. Cross-links were reversed by adding Pronase $(20 \mathrm{mg} / \mathrm{mL})$ 
and incubating the samples for $1 \mathrm{~h}$ at $42^{\circ} \mathrm{C}$ and overnight at $65^{\circ} \mathrm{C}$. DNA was purified using Qiagen minicolumns.

ChIP analysis was performed using real-time PCR with a Corbett Rotorgene system. Input, IP, and NO (no antibody control) samples were all run in triplicate to ensure data accuracy. PCR up to 45 cycles was carried out using the Qiagen QuantiTect SYBR Green PCR Master mix or Quantace SensiMix NoRef DNA Kit. Primers were used at $0.5 \mu \mathrm{M}$ for each experiment. Fluorescent intensities were calculated by

\section{(IP average - NO average)/(Input average).}

For TAP-tagged Ratlp and Sen1p ChIPs (Fig. 4B), IP signals were divided by the relative input and the fold enrichment is expressed relative to the isogenic nontagged strain.

Average data sets are presented. Error bars reflect the SD of average signal obtained between two and four independent experiments.

The coordinates of the PCR primers used are shown in Supplemental Table 3.

\section{TRO analysis}

TRO analysis and probes for rDNA, CYC1, and pTEF:KAN ${ }^{R}(\mathrm{~K} 1)$ were all described previously (Birse et al. 1998; Alen et al. 2002; Morillon et al. 2003; Jones et al. 2007), and locations of the probes used are shown in Figures 1A, 2A, and 4A. Signals were plotted as a percentage of probe 2 for $\mathrm{PDNA}$ or normalized to the probe 1 signal for CYC1 to allow for direct comparison between wild-type and several mutant strains. For the TRO analysis of TAK401 and TAK314 strains, 120-bp-long oligo probes $(2,3,4$, $5,3^{\prime}, 4^{\prime}$, and $5^{\prime}$ ) were used (Supplemental Table 2), and the signals obtained were normalized toward the A content of the probes. For TRO on CYC1 and rDNA representative data are presented based on multiple experiments.

\section{$R T-P C R$}

Standard conditions were used. In outline, total RNA was isolated from logarithmically growing yeast strains after indicated temperature shift times. cDNA was synthesized by RT primed with specific RT primers (p5R and p8-11R in Fig. 3C; p1-6 in Fig. 4C) and PCR-amplified by Taq polymerase for 30 cycles with indicated PCR primers. For both RT-PCR analyses (Figs. 3C, 4C), no genomic DNA contamination was confirmed by $-\mathrm{RT}$ controls. The locations of the RT and PCR primers are shown in Figures 3A and 4A, and their sequences are in Supplemental Table 4.

\section{Acknowledgments}

We are grateful for helpful discussion and encouragement from members of the N.J.P. laboratory. We thank Takehiko Kobayashi for the TAK314 and TAK401 strains, Michel Riva for RPA190 antibody, and Jonathan R. Warner for Reb1 antibody. This work was supported by a Wellcome Trust Programme Grant to N.J.P. H.E.M. is supported by a Scholarship from Boehringer Ingleheim Fonds.

\section{References}

Alen, C., Kent, N.A., Jones, H.S., O'Sullivan, J., Aranda, A., and Proudfoot, N.J. 2002. A role for chromatin remodeling in transcriptional termination by RNA polymerase II. Mol. Cell 10: $1441-1452$.
Archambault, J. and Friesen, J.D. 1993. Genetics of eukaryotic RNA polymerases I, II, and III. Microbiol. Rev. 57: 703-724.

Arigo, J.T., Eyler, D.E., Carroll, K.L., and Corden, J.L. 2006. Termination of cryptic unstable transcripts is directed by yeast RNA-binding proteins Nrd1 and Nab3. Mol. Cell 23: 841851.

Birse, C.E., Minvielle-Sebastia, L., Lee, B.A., Keller, W., and Proudfoot, N.J. 1998. Coupling termination of transcription to messenger RNA maturation in yeast. Science 280: 298 301.

Braglia, P., Percudani, R., and Dieci, G. 2005. Sequence context effects on oligo(dT) termination signal recognition by Saccharomyces cerevisiae RNA polymerase III. J. Biol. Chem. 280: 19551-19562.

Buratowski, S. 2005. Connections between mRNA 3' end processing and transcription termination. Curr. Opin. Cell Biol. 17: 257-261.

Dohmen, R.J., Wu, P., and Varshavsky, A. 1994. Heat-inducible degron: A method for constructing temperature-sensitive mutants. Science 263: 1273-1276.

Dragon, F., Gallagher, J.E., Compagnone-Post, P.A., Mitchell, B.M., Porwancher, K.A., Wehner, K.A., Wormsley, S., Settlage, R.E., Shabanowitz, J., Osheim, Y., et al. 2002. A large nucleolar U3 ribonucleoprotein required for $18 \mathrm{~S}$ ribosomal RNA biogenesis. Nature 417: 967-970.

French, S.L., Osheim, Y.N., Cioci, F., Nomura, M., and Beyer, A.L. 2003. In exponentially growing Saccharomyces cerevisiae cells, rRNA synthesis is determined by the summed RNA polymerase I loading rate rather than by the number of active genes. Mol. Cell. Biol. 23: 1558-1568.

Ganley, A.R., Hayashi, K., Horiuchi, T., and Kobayashi, T. 2005. Identifying gene-independent noncoding functional elements in the yeast ribosomal DNA by phylogenetic footprinting. Proc. Natl. Acad. Sci. 102: 11787-11792.

Grummt, I., Rosenbauer, H., Niedermeyer, I., Maier, U., and Ohrlein, A. 1986. A repeated 18 bp sequence motif in the mouse rDNA spacer mediates binding of a nuclear factor and transcription termination. Cell 45: 837-846.

Houseley, J., Kotovic, K., El Hage, A., and Tollervey, D. 2007. Trf4 targets ncRNAs from telomeric and rDNA spacer regions and functions in rDNA copy number control. EMBO $J$. 26: 4996-5006.

Jansa, P. and Grummt, I. 1999. Mechanism of transcription termination: PTRF interacts with the largest subunit of RNA polymerase I and dissociates paused transcription complexes from yeast and mouse. Mol. Gen. Genet. 262: 508-514.

Jansa, P., Mason, S.W., Hoffmann-Rohrer, U., and Grummt, I. 1998. Cloning and functional characterization of PTRF, a novel protein which induces dissociation of paused ternary transcription complexes. EMBO J. 17: 2855-2864.

Jeong, S.W., Lang, W.H., and Reeder, R.H. 1995. The release element of the yeast polymerase I transcription terminator can function independently of Reblp. Mol. Cell. Biol. 15: 5929-5936.

Jones, H.S., Kawauchi, J., Braglia, P., Alen, C.M., Kent, N.A., and Proudfoot, N.J. 2007. RNA polymerase I in yeast transcribes dynamic nucleosomal rDNA. Nat. Struct. Mol. Biol. 14: 123-130.

Kaneko, S., Rozenblatt-Rosen, O., Meyerson, M., and Manley, J.L. 2007. The multifunctional protein $\mathrm{p} 54 \mathrm{nrb} / \mathrm{PSF}$ recruits the exonuclease XRN2 to facilitate pre-mRNA 3' processing and transcription termination. Genes \& Dev. 21: 1779-1789.

Keogh, M.C. and Buratowski, S. 2004. Using chromatin immunoprecipitation to map cotranscriptional mRNA processing in Saccharomyces cerevisiae. Methods Mol. Biol. 257: 1-16.

Kim, M., Ahn, S.H., Krogan, N.J., Greenblatt, J.F., and Bura- 
towski, S. 2004a. Transitions in RNA polymerase II elongation complexes at the $3^{\prime}$ ends of genes. EMBO J. 23: 354-364.

Kim, M., Krogan, N.J., Vasiljeva, L., Rando, O.J., Nedea, E., Greenblatt, J.F., and Buratowski, S. 2004b. The yeast Rat1 exonuclease promotes transcription termination by RNA polymerase II. Nature 432: 517-522.

Kim, M., Vasiljeva, L., Rando, O.J., Zhelkovsky, A., Moore, C., and Buratowski, S. 2006. Distinct pathways for snoRNA and mRNA termination. Mol. Cell 24: 723-734.

Kufel, J., Dichtl, B., and Tollervey, D. 1999. Yeast Rntlp is required for cleavage of the pre-ribosomal RNA in the 3' ETS but not the 5' ETS. RNA 5: 909-917.

Kuhn, A., Bartsch, I., and Grummt, I. 1990. Specific interaction of the murine transcription termination factor TTF I with class-I RNA polymerases. Nature 344: $559-562$.

Kuhn, C.D., Geiger, S.R., Baumli, S., Gartmann, M., Gerber, J., Jennebach, S., Mielke, T., Tschochner, H., Beckmann, R., and Cramer, P. 2007. Functional architecture of RNA polymerase I. Cell 131: 1260-1272.

Kulkens, T., van Heerikhuizen, H., Klootwijk, J., Oliemans, J., and Planta, R.J. 1989. A yeast ribosomal DNA-binding protein that binds to the rDNA enhancer and also close to the site of Pol I transcription initiation is not important for enhancer functioning. Curr. Genet. 16: 351-359.

Lang, W.H. and Reeder, R.H. 1993. The REB1 site is an essential component of a terminator for RNA polymerase I in Saccharomyces cerevisiae. Mol. Cell. Biol. 13: 649-658.

Lang, W.H., Morrow, B.E., Ju, Q., Warner, J.R., and Reeder, R.H. 1994. A model for transcription termination by RNA polymerase I. Cell 79: 527-534.

Luo, W., Johnson, A.W., and Bentley, D.L. 2006. The role of Rat1 in coupling mRNA 3 '-end processing to transcription termination: Implications for a unified allosteric-torpedo model. Genes \& Dev. 20: 954-965.

Morillon, A., Karabetsou, N., O'Sullivan, J., Kent, N., Proudfoot, N., and Mellor, J. 2003. Isw1 chromatin remodeling ATPase coordinates transcription elongation and termination by RNA polymerase II. Cell 115: 425-435.

Morrow, B.E., Johnson, S.P., and Warner, J.R. 1989. Proteins that bind to the yeast rDNA enhancer. J. Biol. Chem. 264: 90619068.

Nemeth, A., Guibert, S., Tiwari, V.K., Ohlsson, R., and Langst, G. 2008. Epigenetic regulation of TTF-I mediated promoterterminator interactions of rRNA genes. $E M B O \mathrm{~J}$. (in press).

Orphanides, G. and Reinberg, D. 2002. A unified theory of gene expression. Cell 108: 439-451.

O'Sullivan, J.M., Tan-Wong, S.M., Morillon, A., Lee, B., Coles, J., Mellor, J., and Proudfoot, N.J. 2004. Gene loops juxtapose promoters and terminators in yeast. Nat. Genet. 36: 10141018

Prescott, E.M., Osheim, Y.N., Jones, H.S., Alen, C.M., Roan, J.G., Reeder, R.H., Beyer, A.L., and Proudfoot, N.J. 2004. Transcriptional termination by RNA polymerase I requires the small subunit Rpa12p. Proc. Natl. Acad. Sci. 101: 60686073 .

Proudfoot, N. 2004. New perspectives on connecting messenger RNA 3' end formation to transcription. Curr. Opin. Cell Biol. 16: 272-278.

Reeder, R.H., Guevara, P., and Roan, J.G. 1999. Saccharomyces cerevisiae RNA polymerase I terminates transcription at the Reb1 terminator in vivo. Mol. Cell. Biol. 19: 7369-7376.

Steinmetz, E.J., Conrad, N.K., Brow, D.A., and Corden, J.L. 2001. RNA-binding protein $\mathrm{Nrdl}$ directs poly(A)-independent 3 '-end formation of RNA polymerase II transcripts. $\mathrm{Na}$ ture 413: 327-331.

Steinmetz, E.J., Warren, C.L., Kuehner, J.N., Panbehi, B., Ansari,
A.Z., and Brow, D.A. 2006. Genome-wide distribution of yeast RNA polymerase II and its control by Sen 1 helicase. Mol. Cell 24: 735-746.

Tollervey, D. 2004. Molecular biology: Termination by torpedo. Nature 432: 456-457.

Ursic, D., Himmel, K.L., Gurley, K.A., Webb, F., and Culbertson, M.R. 1997. The yeast SEN1 gene is required for the processing of diverse RNA classes. Nucleic Acids Res. 25: 4778-4785.

Ursic, D., Chinchilla, K., Finkel, J.S., and Culbertson, M.R 2004. Multiple protein/protein and protein/RNA interactions suggest roles for yeast DNA/RNA helicase Sen1p in transcription, transcription-coupled DNA repair and RNA processing. Nucleic Acids Res. 32: 2441-2452.

Vasiljeva, L., Kim, M., Terzi, N., Soares, L.M., and Buratowski, S. 2008. Transcription termination and RNA degradation contribute to silencing of RNA polymerase II transcription within heterochromatin. Mol. Cell 29: 313-323.

Wai, H., Johzuka, K., Vu, L., Eliason, K., Kobayashi, T., Horiuchi, T., and Nomura, M. 2001. Yeast RNA polymerase I enhancer is dispensable for transcription of the chromosomal rRNA gene and cell growth, and its apparent transcription enhancement from ectopic promoters requires Fob1 protein. Mol. Cell. Biol. 21: 5541-5553.

West, S., Gromak, N., and Proudfoot, N.J. 2004. Human $5^{\prime} \rightarrow 3^{\prime}$ exonuclease Xrn2 promotes transcription termination at cotranscriptional cleavage sites. Nature 432: 522-525. 


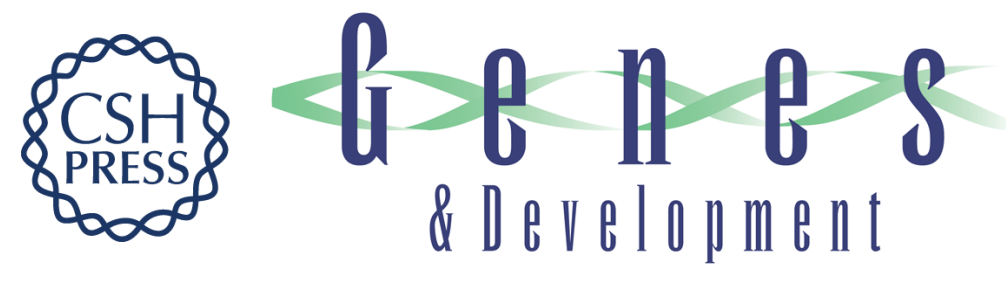

\section{Budding yeast RNA polymerases I and II employ parallel mechanisms of transcriptional termination}

Junya Kawauchi, Hannah Mischo, Priscilla Braglia, et al.

Genes Dev. 2008, 22:

Access the most recent version at doi:10.1101/gad.463408

Supplemental http://genesdev.cshlp.org/content/suppl/2008/03/27/22.8.1082.DC1
Material

References This article cites 43 articles, 17 of which can be accessed free at: http://genesdev.cshlp.org/content/22/8/1082.full.html\#ref-list-1

License Freely available online through the Genes \& Development Open Access option.

Email Alerting Receive free email alerts when new articles cite this article - sign up in the box at the top Service right corner of the article or click here.

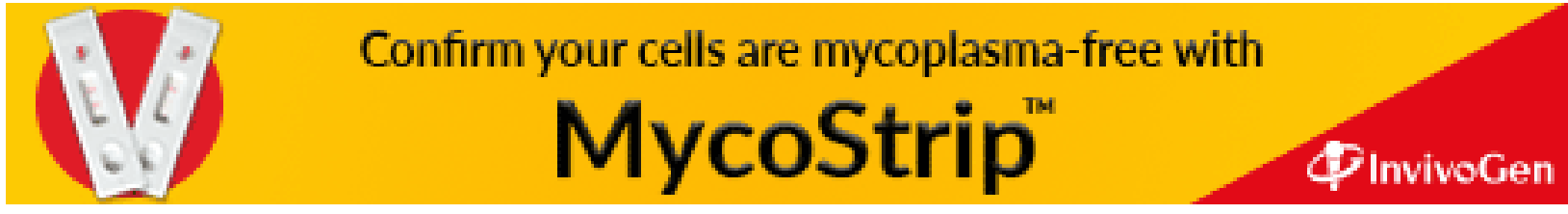

